\title{
Roles of DMP1 Processing in Osteogenesis, Dentinogenesis and Chondrogenesis
}

\author{
Yao Sun ${ }^{a, b}$ Li Chen $^{c}$ Su Ma ${ }^{d}$ Jin Zhou ${ }^{e}$ Hua Zhang ${ }^{a}$ Jian Q. Feng ${ }^{a}$ \\ Chunlin Qin ${ }^{\mathrm{a}}$ \\ ${ }^{a}$ Department of Biomedical Sciences, Baylor College of Dentistry, Texas A\&M Health Science Center, Dallas, Tex., USA; \\ Departments of ${ }^{b}$ Dental Implants, ' Endodontics, and d Periodontics, School of Stomatology, Harbin Medical

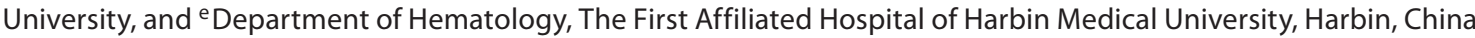

\section{Key Words}

Dentin matrix protein $1 \cdot$ Proteolytic processing $\cdot$

Osteogenesis $\cdot$ Dentinogenesis $\cdot$ Chondrogenesis

\begin{abstract}
Dentin matrix protein 1 (DMP1) is an acidic protein that plays critical roles in osteogenesis and dentinogenesis. Protein chemistry studies have demonstrated that DMP1 primarily exists as processed $\mathrm{NH}_{2}$ - and $\mathrm{COOH}$-terminal fragments in the extracellular matrix of bone and dentin. Our earlier work showed that the substitution of $\mathrm{Asp}^{213}$ (a residue at a cleavage site) by $\mathrm{Ala}^{213}$ blocks the processing of mouse DMP1 in vitro. Recently, we generated transgenic mice expressing this mutant DMP1 (designated 'D213A-DMP1'). By crossbreeding these transgenic mice with Dmp1-knockout (Dmp1-KO) mice, we obtained mice expressing the D213ADMP1 transgene in the Dmp1-null background (named 'Dmp1-KO/D213A-Tg' mice). In this study, we analyzed the long bone, mandible, dentin, and cartilage of Dmp1$\mathrm{KO} / \mathrm{D} 213 \mathrm{~A}-\mathrm{Tg}$ mice in comparison with wild-type, Dmp1-KO, and Dmp1-KO mice expressing the normal DMP1 transgene (Dmp1-KO/normal-Tg). Our results showed that D213A-DMP1 was barely cleaved in the dentin matrix of Dmp1-KO/D213A-
\end{abstract}

Yao Sun and Li Chen contributed equally to this work.
Tg mice and the expression of D213A-DMP1 failed to rescue the developmental defects in Dmp1-null mice. Interestingly, enlarged growth plates and condylar cartilages were observed in Dmp1-KO/D213A-Tg mice, indicating a potential role of the full-length form of DMP1 in chondrogenesis.

Copyright $\odot 2011$ S. Karger AG, Basel

\section{Introduction}

Dentin matrix protein 1 (DMP1) was first identified using a rat odontoblast mRNA library [George et al., 1993] which was later shown to be also expressed in bone and other tissues [D'Souza et al., 1997; Hirst et al., 1997; MacDougall et al., 1998; Qin et al., 2007]. Loss of DMP1

\begin{tabular}{ll} 
Abbreviations used in this paper \\
\hline BMP-1 & bone morphogenetic protein-1 \\
DMP1 & dentin matrix protein 1 \\
HE & hematoxylin and eosin \\
IHC & immunohistochemistry \\
ISH & in situ hybridization \\
KO & knockout \\
NCPs & noncollagenous protein \\
WT & wild type
\end{tabular}


leads to osteomalacia/rickets and dentin hypomineralization, indicating that DMP1 plays important roles in osteogenesis and dentinogenesis [Ye et al., 2004, 2005; Feng et al., 2006].

In the extracellular matrix of bone and dentin, DMP1 mainly occurs as proteolytically processed fragments originating from the $\mathrm{NH}_{2}$-terminal and $\mathrm{COOH}$-terminal regions of the DMP1 amino acid sequence, respectively. The $\mathrm{NH}_{2}$-terminal fragment of DMP1 (designated as 'DMP1-N') exists in 2 forms, i.e. the $37-\mathrm{kDa}$ core protein form and the proteoglycan form (designated as DMP1$\mathrm{PG}$ ), while the $\mathrm{COOH}$-terminal fragment (designated as 'DMP1-C') is present as the $57-\mathrm{kDa}$ fragment [Qin et al., 2003, 2006]. The full-length form of DMP1 has been detected at a lower level in the extracellular matrix of bone and dentin [Huang et al., 2008] and at a relatively high level in articular cartilage [Sun et al., 2010a, b].

In vitro mineralization studies have shown that DMP1-C and DMP1-N fragments promote the nucleation of hydroxyapatite crystals while full-length DMP1 and DMP1-PG inhibit their growth [Tartaix et al., 2004; Gericke et al., 2010]. Studies have shown that the localization of DMP1-N is different from that of DMP1-C in tooth and long bone [Maciejewska et al., 2009]. Our previous work showed that rat DMP1 is proteolytically processed into DMP1-N and DMP1-C at 4 peptide bonds: $\mathrm{Phe}^{189}-\mathrm{Asp}^{190}, \mathrm{Ser}^{196}-\mathrm{Asp}^{197}, \mathrm{Ser}^{233}-\mathrm{Asp}^{234}$, and $\mathrm{Gln}^{237}$ Asp $^{238}$ [Qin et al., 2003]. An in vitro study demonstrated that bone morphogenetic protein-1 (BMP-1)/tolloid-like proteinases cleave the full-length form of rat DMP1 at peptide bond Ser ${ }^{196}$-Asp ${ }^{197}$ [Tartaix et al., 2004]. Moreover, the substitution of $\mathrm{Asp}^{213}$ in mouse DMP1 (corresponding to $\mathrm{Asp}^{197}$ in rat DMP1) effectively blocked the proteolytic processing of mouse DMP1 in eukaryotic cells [Peng et al., 2009]. Recently, we generated transgenic mice expressing the D213A-DMP1 transgene in a Dmp1-null background (named 'Dmp1-KO/D213A-Tg' mice), and D213A-DMP1 was barely cleaved in bone [Sun et al., 2010c]. In this report, we analyze the phenotypic changes in the bone, dentin and cartilage expressing this mutant D213A-DMP1 in the Dmp1-null background.

\section{Materials and Methods}

Generation of Transgenic Mice

The details of the generation of Dmp1-knockout (Dmp1-KO) mice, Dmp1-KO/D213A-Tg mice, and Dmp1-KO mice expressing the normal DMP1 transgene (Dmp1-KO/normal- Tg) have been described in previous publications [Feng et al., 2003; Lu et al., 2007, 2010; Sun et al., 2010c].
Detection of DMP-Related Products in Dentin Matrix and Digestion of DMP1 by BMP-1

Noncollagenous proteins (NCPs) were extracted from the dentin matrix of 6-week-old wild-type (WT), Dmp1-KO, Dmp1$\mathrm{KO} / \mathrm{D} 213 \mathrm{~A}-\mathrm{Tg}$, and Dmp1-KO/normal-Tg mice. The NCPs, including DMP1, were analyzed by Stains-All staining and Western immunoblotting [Sun et al., 2010c]. Stains-All staining was employed to examine full-length DMP1 and/or its processed fragments. Western immunoblotting was carried out to confirm the presence/absence of DMP1. To test whether BMP-1 cleaves DMP1 isolated from dentin, normal DMP1 (from Dmp1-KO/normal-Tg mice) and D213A-DMP1 (from Dmp1-KO/D213A-Tg mice) were incubated with recombinant human BMP-1 (R\&D Systems, Minneapolis, Minn., USA) as previously described [Steiglitz et al., 2004]. For Western immunoblotting, the polyclonal anti-DMP1C-857 antibody [Maciejewska et al., 2009] was used.

\section{X-Ray Analyses}

Femurs were dissected from 1-year-old WT, Dmp1-KO, Dmp1$\mathrm{KO} / \mathrm{D} 213 \mathrm{~A}-\mathrm{Tg}$, and Dmp1-KO/normal-Tg mice. These specimens were analyzed by high-resolution X-ray radiography (piXarray 100; Micro Photonics) at $5 \mathrm{~s} / 30 \mathrm{kV}$.

\section{Histology, Immunohistochemistry, and in situ Hybridization} Analyses

Hematoxylin and eosin (HE) staining was used to evaluate the phenotypic changes in the long bone, incisors, and cartilage of those mice. Immunohistochemistry (IHC) and in situ hybridization (ISH) were used to evaluate the expression of normal DMP1/ D213A-DMP1 in the long bone, incisors, and cartilage of those mice. For IHC, the monoclonal anti-DMP1-C-8G10.3 antibody was used [Baba et al., 2004]. ISH was performed as previously described [Lu et al., 2007].

\section{Results}

Identification of DMP1 in Dentin Extracts and Its Digestion by BMP-1

Stains-All staining showed that the dentin of Dmp1$\mathrm{KO} / \mathrm{D} 213 \mathrm{~A}-\mathrm{Tg}$ mice had significant amounts of fulllength DMP1 and a negligible quantity of DMP1 fragments (fig. 1a). The enzyme digestion experiments showed that the normal full-length DMP1 isolated from Dmp1-KO/normal-Tg mice was cleaved by BMP-1, while the mutant D213A-DMP1 isolated from the dentin of Dmp1-KO/D213A-Tg mice was hard to cleave (fig. $1 \mathrm{~b}$ ).

\section{$X$-Ray Analyses}

The femurs of Dmp1-KO/D213A-Tg mice were shorter and had a lower density than those of WT or Dmp1$\mathrm{KO} /$ normal-Tg mice. Thus, the bone development defects in Dmp1-KO/D213A-Tg mice remained similar to those in Dmp1-KO mice (fig. 1c). 


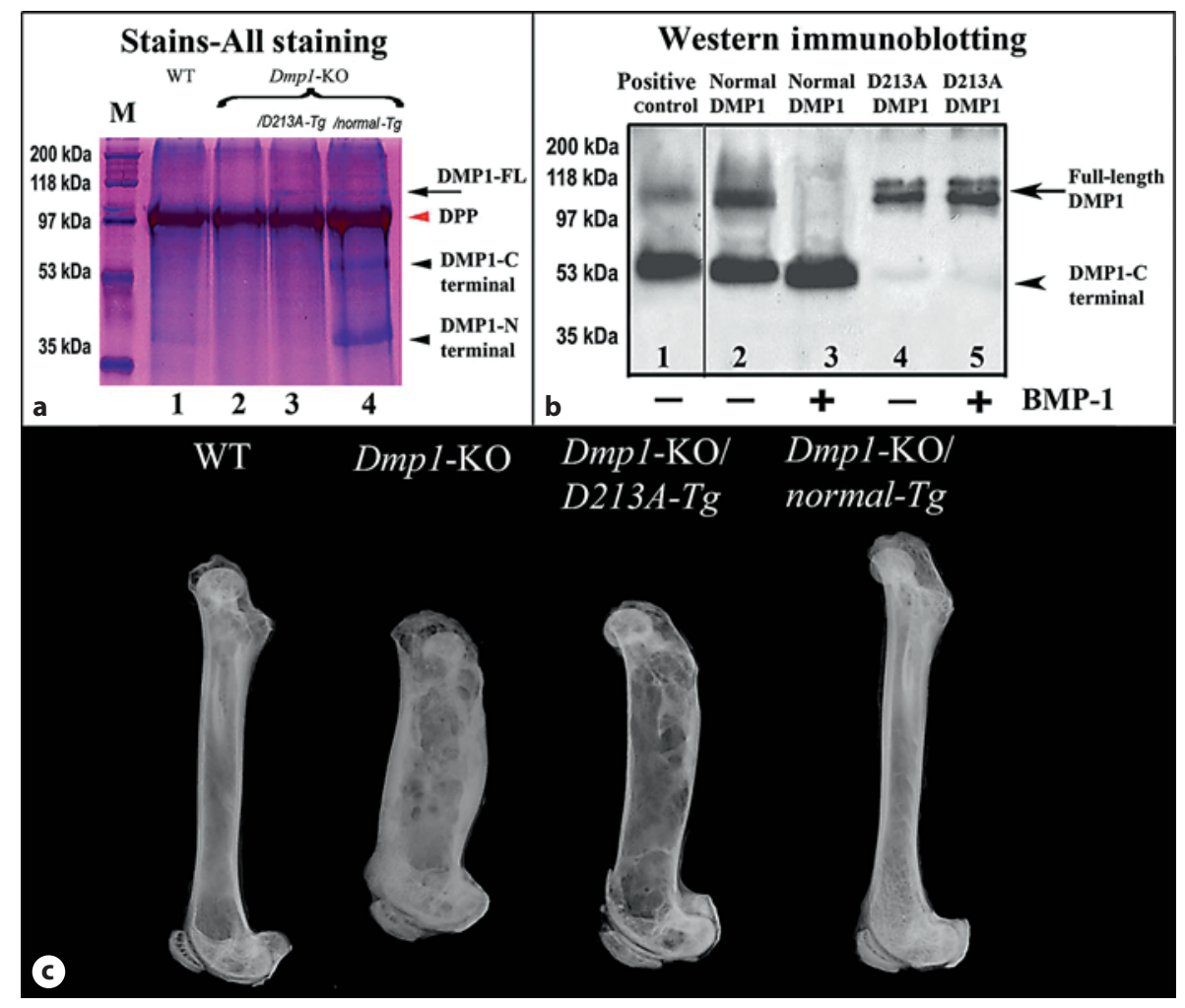

Fig. 1. Detection of DMP1 in dentin extracts, digestion of DMP1 by BMP-1, and X-ray images of femurs. a NCPs were extracted from the dentin of 6-week-old WT (lane 1), Dmp1-KO (lane 2), Dmp1-KO/D213A-Tg (lane 3), and Dmp1-KO/normal-Tg (lane 4) mice. Stains-All staining was performed to detect DMP1-related components. Full-length DMP1 (black arrow) was clearly observed in the dentin extracts from Dmp1-KO/D213A-Tg mice (lane 3), while DMP1-N and DMP1-C fragments (black arrowheads) were seen in the dentin extracts from WT and Dmp1-KO/ normal-Tg mice (lanes 1 and 4). DPP (strong blue bands signalized by a red arrowhead). $\mathbf{b}$ Digestion of DMP1 by BMP-1. The digestion products were analyzed by Western immunoblotting using the anti-DMP1-C-857 antibody. Lane 1 was $0.2 \mu \mathrm{g}$ purified DMP1 extracted from rat dentin (positive control). The normal DMP1 (lane 3, from the dentin of Dmp1-KO/normal-Tg) and D213ADMP1 (lane 5, from Dmp1-KO/D213A-Tg) were incubated with BMP-1. Note that most of the full-length form of normal DMP1 was processed into fragments (lane 3) by BMP-1 while D213ADMP1 was not (lane 5). Also note that the full-length form of D213A-DMP1 appeared as doublets; at this point, we are unsure as to why it occurred as 2 bands. c X-ray images of the femurs of 1-year-old WT, Dmp1-KO, Dmp1-KO/D213A-Tg, and Dmp1$\mathrm{KO} /$ normal- $\mathrm{Tg}$ mice. Note that the femurs of Dmp1-KO or Dmp1$\mathrm{KO} / \mathrm{D} 213 \mathrm{~A}-\mathrm{Tg}$ mice were shorter.

\section{Histological Analyses of Bone and Dentin}

HE staining demonstrated that the bone and dentin phenotypes of Dmp1-KO/D213A-Tg mice were different from those of WT mice (fig. 2). Compared to those of WT mice of the same age, the femurs of 1-year-old Dmp1$\mathrm{KO} / D 213 A-\mathrm{Tg}$ mice were shorter; the metaphysis region was disorganized, and more osteoid was observed in the cortical bone area. In the incisors, the predentin in Dmp1-KO/D213A-Tg mice was wider than that in WT mice (note the distance between the 2 black arrows in fig. $2 g$ and $h$ ).

\section{Phenotypic Changes in the Cartilage of Dmp1-KO/}

D213A-Tg Mice and Expression of DMP1 in the

Growth Plate of Tibia

The growth plate of $\mathrm{Dmp1}-\mathrm{KO} / \mathrm{D} 213 \mathrm{~A}-\mathrm{Tg}$ mice was remarkably thicker than that of WT mice. More layers of chondrocytes were observed in the hypertrophic layer and proliferative layer in the enlarged growth plate of Dmp1-KO/D213A-Tg mice. The organization of the growth plate in Dmp1-KO/D213A-Tg mice was better than in Dmp1-KO mice. The condylar cartilage of the mandibles of Dmp1-KO/D213A-Tg mice was also enlarged (fig. 3). In the growth plate, the distribution of DMP1 in Dmp1-KO/D213A-Tg mice was different from that in WT 

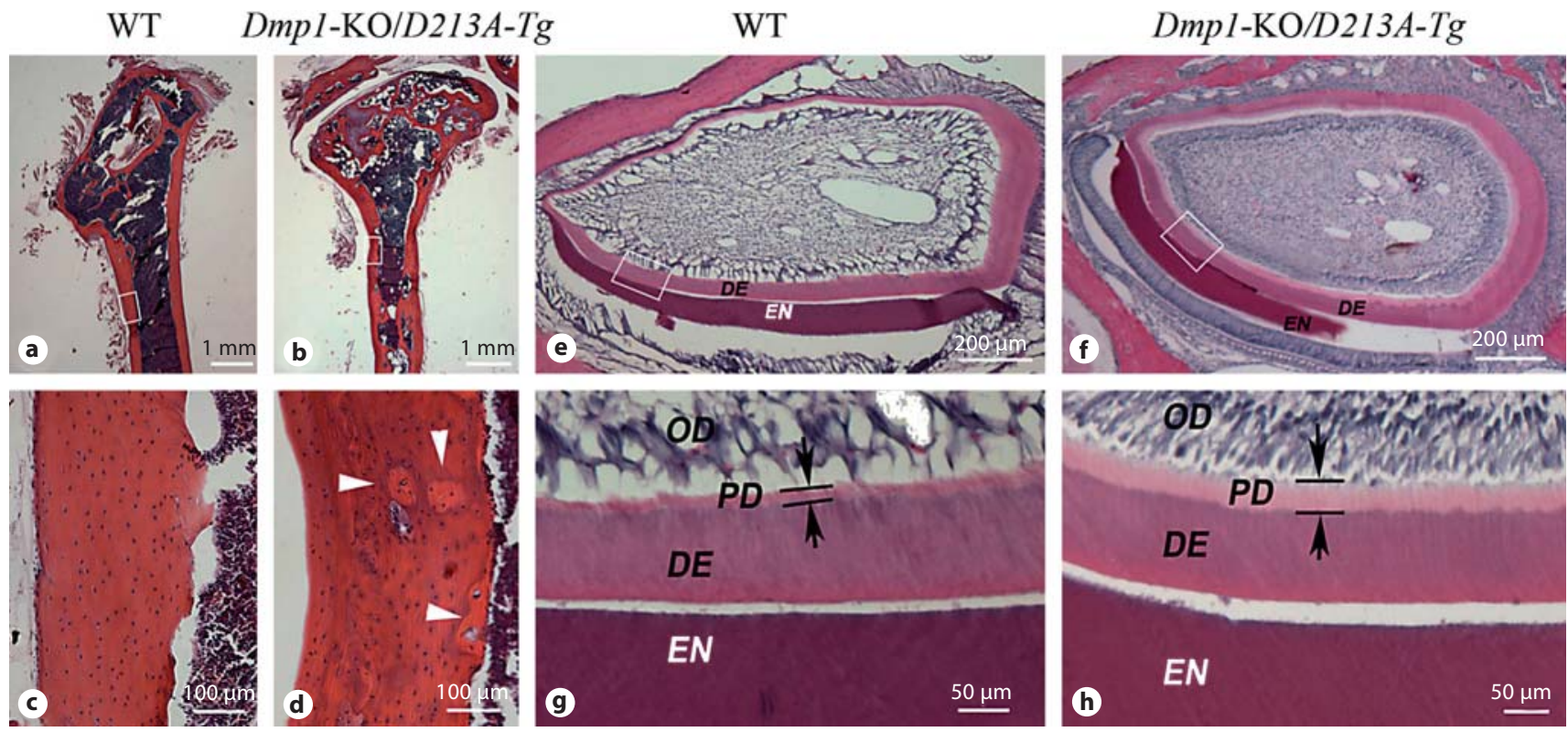

Fig. 2. Histological analyses. a, b HE staining of the femurs of 1-year-old WT and Dmp1-KO/D213A-Tg mice. c, d Enlarged views of the cortical bone (insets in $\mathbf{a}, \mathbf{b})$ from the diaphysis region. e, $\mathbf{f}$ The cross sections of the incisors of 1-year-old WT and Dmp1-KO/D213A-Tg mice were analyzed. $\mathbf{g}, \mathbf{h}$ Enlarged views of the predentin/dentin region (insets in $\mathbf{e}, \mathbf{f}$ ). Compared to WT mice, the femurs of 1-year-old Dmp1-KO/D213A-Tg mice were shorter and the metaphysis region was disorganized, with more osteoid in the cortical bone area. In the incisors, the predentin of Dmp1-KO/D213A-Tg mice was wider than that of WT mice. DE = Dentin; $\mathrm{OD}=$ odontoblast $\mathrm{PD}=$ predentin; $\mathrm{EN}=$ enamel. mice. In WT mice, signals of normal DMP1 mRNA and protein were mainly observed in cells of the hypertrophic layer, while in Dmp1-KO/D213A-Tg mice signals of DMP1 were primarily found in cells of the proliferative layer (fig. 3).

\section{Discussion}

Previously we hypothesized that proteolytic processing of DMP1 may be essential for the biological functions of this protein in biomineralization [Qin et al., 2007]. The results of this study show that the bone and dentin phenotypes of 1-year-old Dmp1-KO/D213A-Tg mice were similar to those of Dmp1-KO mice. In addition, the expression of this D213A-DMP1 failed to rescue the phenotypic changes in the long bone of 6-week-old Dmp1-KO mice [Sun et al., 2010c], but the normal DMP1 could [Lu et al., 2010; Sun et al., 2010c]. All of these results provide strong support to our belief that DMP1 processing is essential to the formation of bone and dentin. The present study also shows that BMP-1 is the proteinase responsible for the proteolytic processing of DMP1 at the $\mathrm{NH}_{2}$-terminus of $\mathrm{Asp}^{213}$ in mouse DMP1 [Steiglitz et al., 2004].
The growth plate and condylar cartilage in Dmp1$\mathrm{KO} / \mathrm{D} 213 \mathrm{~A}-\mathrm{Tg}$ mice were remarkably thicker than those in WT and Dmp1-KO mice. Previous studies reported an enlargement of the growth plate cartilage in Dmp1-KO mice, which was believed to be a result of the decrease in serum phosphate level [Feng et al., 2006]. It has been shown that the serum phosphate level in Dmp1-KO/ D213A-Tg mice is higher than in Dmp1-KO mice [Sun et al., 2010c], whereas the growth plate and condylar cartilage in Dmp1$\mathrm{KO} / \mathrm{D} 213 \mathrm{~A}-\mathrm{Tg}$ mice is even thicker than in Dmp1-KO mice. Thus, a lower level of phosphate could not well explain the extraordinary thickening of the cartilage in Dmp1$\mathrm{KO} / \mathrm{D} 213 \mathrm{~A}-\mathrm{Tg}$ mice at the age of 6 weeks. It has also recently become known that, compared with WT mice, the major phenotypic change in the growth plate of 6-week-old or older Dmp1-KO mice is disorganization but not enlargement [Lu et al., 2010; Sun et al., 2010c]. Combined with the finding that a large amount of full-length DMP1 exists in cartilage matrix [Sun et al., 2010a, b], we speculate that the full-length form of DMP1 may play a certain role in chondrogenesis. The observation that the cellular organization of cartilage in Dmp1-KO/D213A-Tg mice was much better than in Dmp1-KO mice also supports this speculation. Clearly, further investigations are warranted to evaluate 

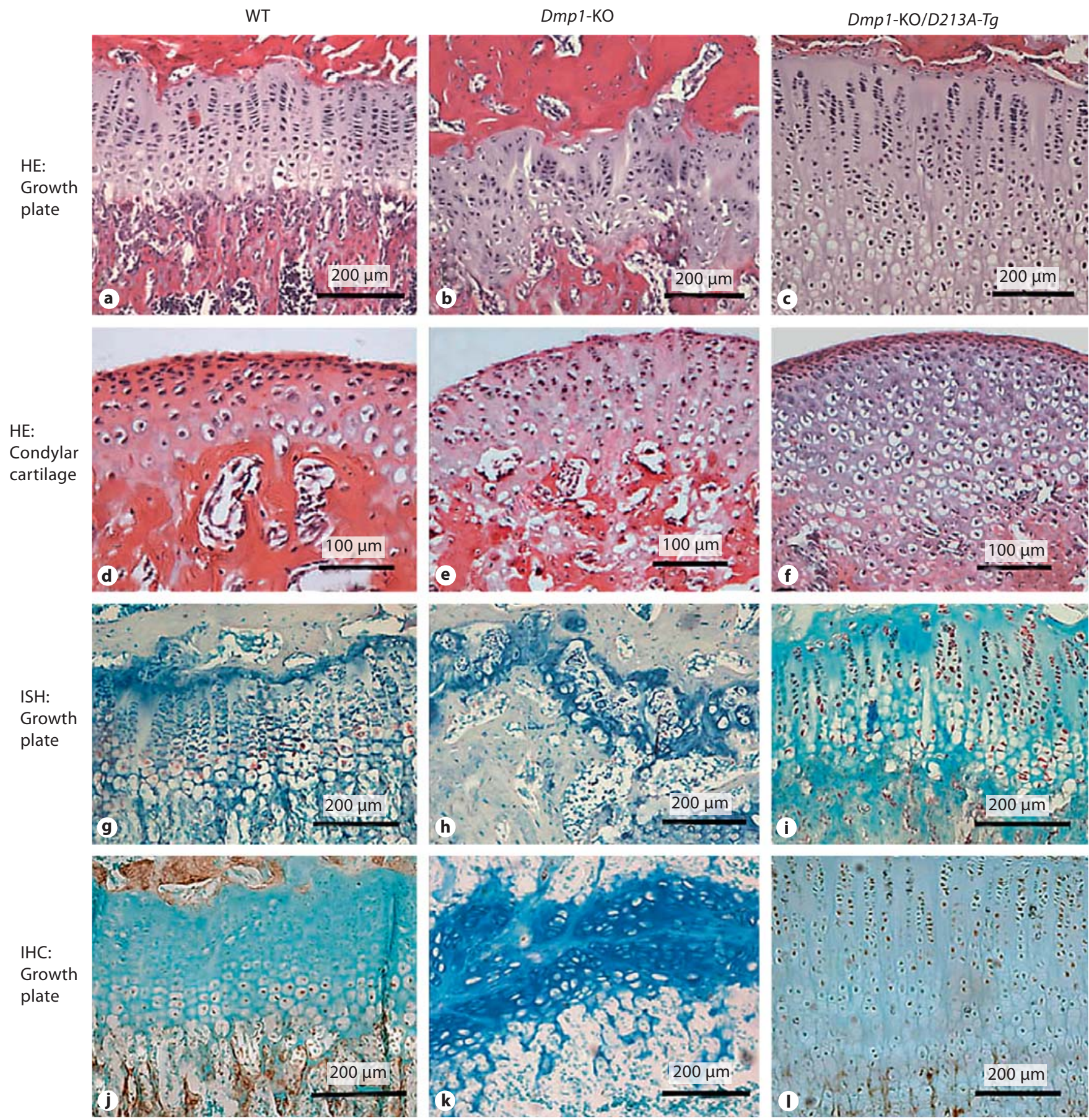

Fig. 3. Phenotypic changes in the cartilage of $D m p 1-\mathrm{KO} / \mathrm{D} 213 \mathrm{~A}-\mathrm{Tg}$ mice and expression of DMP1 in the growth plate of the tibia. All samples were from 6-week-old mice. a-c The growth plate of Dmp1-KO mice was disorganized, and the cells in the Dmp1-KO growth plate lost their columnar arrangement. The growth plate of Dmp1-KO/D213A-Tg mice was thicker than that of either WT or Dmp1-KO mice, and the organization of the growth plate in Dmp1-KO/D213A-Tg mice was better than that in Dmp1-KO mice. d-f HE staining for the mandibular condylar cartilage from WT, Dmp1-KO, and Dmp1-KO/D213A-Tg mice. Similar to the findings in the growth plate, the condylar cartilage of $\mathrm{Dmp1}-\mathrm{KO} / \mathrm{D} 213 \mathrm{~A}-\mathrm{Tg}$ mice was thicker than that of WT or Dmp1-KO mice. g-i ISH demonstrated positive signals of DMP1 in the chondrocytes of both WT and Dmp1-KO/D213A-Tg mice. In WT mice, the positive signals in the growth plate were mainly detected in the hypertrophic layers, while in Dmp1-KO/D213A-Tg mice the signals were mainly present in the proliferative layer. $\mathbf{j}-\mathbf{I}$ IHC staining of growth plates revealed a distribution pattern of DMP1 protein similar to the results obtained by ISH. 
the role of full-length DMP1 in the development of cartilage. As articular cartilage, mandibular condylar cartilage expresses both type I and type II collagen. Thus, it is understandable that the D213A-DMP1 transgene (driven by a type I collagen promoter) was expressed in condylar cartilage and might contribute to the formation of articular cartilage. However, we do not know why the D213A-DMP1 transgene was also expressed in the growth plate, which normally does not express type I collagen.

In summary, we strongly believe that the proteolytic processing of DMP1 is essential to its functions in mineralized and cartilaginous tissues. We further envision that DMP1 fragments are functional forms in the mineralization of bone and dentin [Gericke et al., 2010], while the uncleaved full-length DMP1 may play a role in the development of cartilage.

\section{Acknowledgements}

This work was supported by NIH Grant DE 005092 (to C.Q.), Department of Science and Technology of Heilongiiang Province of China - Gongguan Project Grant GC09C412-1, and Department of Education of Heilongjiang Province of China - Overseas Scholar Grant 1155h013.

\section{References}

Baba, O., C. Qin, J.C. Brunn, J.N. Wygant, B.W. Lu, Y., L. Ye, S. Yu, S. Zhang, Y. Xie, M.D. McKee, McIntyre, W.T. Butler (2004) Colocalization of dentin matrix protein 1 and dentin sialoprotein at late stages of rat molar development. Matrix Biol 23: 371-379.

D'Souza, R.N., A. Cavender, G. Sunavala, J. Alvarez, T. Ohshima, A.B. Kulkarni, M. MacDougall (1997) Gene expression patterns of murine dentin matrix protein 1 (Dmp1) and dentin sialophosphoprotein (DSPP) suggest distinct developmental functions in vivo. J Bone Miner Res 12: 2040-2049.

Feng, J.Q., H. Huang, Y. Lu, L. Ye, Y. Xie, T.W. Tsutsui, T. Kunieda, T. Castranio, G. Scott, L.B. Bonewald, Y. Mishina (2003) The Dentin matrix protein 1 (Dmp1) is specifically expressed in mineralized, but not soft, tissues during development. J Dent Res 82: 776-780.

Feng, J.Q., L.M. Ward, S. Liu, Y. Lu, Y. Xie, B. Yuan, X. Yu, F. Rauch, S.I. Davis, S. Zhang, H. Rios, M.K. Drezner, L.D. Quarles, L.F. Bonewald, K.E. White (2006) Loss of DMP1 causes rickets and osteomalacia and identifies a role for osteocytes in mineral metabolism. Nat Genet 38: 1310-1315.

George, A., B. Sabsay, P.A. Simonian, A. Veis (1993) Characterization of a novel dentin matrix acidic phosphoprotein: implications for induction of biomineralization. J Biol Chem 268: 12624-12630.

Gericke, A., C. Qin, Y. Sun, R. Redfern, D. Redfern, Y. Fujimoto, H. Taleb, W.T. Butler, A.L. Boskey (2010) J Dent Res 89: 355-359.

Hirst, K.L., D. Simmons, J. Feng, H. Aplin, M.J. Dixon, M. MacDougall (1997) Elucidation of the sequence and the genomic organization of the human dentin matrix acidic phosphoprotein 1 (DMP1) gene: exclusion of the locus from a causative role in the pathogenesis of dentinogenesis imperfecta type II. Genomics 42: 38-45.

-Huang B., I. Maciejewska, Y. Sun, T. Peng, D. Qin, Y. Lu, L. Bonewald, W.T. Butler, J. Feng, C. Qin (2008) Identification of full-length dentin matrix protein 1 in dentin and bone. Calcif Tissue Int 82: 401-410. Y.C. Li, J. Kong, J.D. Eick, S.L. Dallas, J.Q. Feng (2007) Rescue of odontogenesis in Dmp1-deficient mice by targeted re-expression of DMP1 reveals roles for DMP1 in early odontogenesis and dentin apposition in vivo. Dev Biol 303: 191-201.

Lu, Y., B. Yuan, C. Qin, Z. Cao, Y. Xie, S. Dallas, M.D. McKee, M.K. Drezner, L.F. Bonewald, Jian Q. Feng (2010) The biological function of DMP1 in osteocyte maturation is mediated by its $57-\mathrm{kDa}$ C-terminal fragment. J Bone Miner Res, in press.

MacDougall, M., T.T. Gu, X. Luan, D. Simmons, J. Chen (1998) Identification of a novel isoform of mouse dentin matrix protein 1: spatial expression in mineralized tissues. J Bone Miner Res 13: 422-431.

Maciejewska, I., C. Cowan, K. Svoboda, W.T. Butler, R. D'Souza, C. Qin (2009) The $\mathrm{NH}_{2-}$ terminal and $\mathrm{COOH}$-terminal fragments of dentin matrix protein 1 (DMP1) localize differently in the compartments of dentin and growth plate of bone. J Histochem Cytochem 57: 155-166.

Peng, T., B. Huang, Y. Sun, Y. Lu, L. Bonewald, S. Chen, W.T. Butler, J.Q. Feng, R.N. D'Souza, C. Qin (2009) Blocking of proteolytic processing and deletion of glycosaminoglycan side chain of mouse DMP1 by substituting critical amino acid residues. Cells Tissues Organs 189: 192-197.

Qin, C., J.C. Brunn, R.G. Cook, R.S. Orkiszewski, J.P. Malone, A. Veis, W.T. Butler (2003) Evidence for the proteolytic processing of dentin matrix protein 1: identification and characterization of processed fragments and cleavage sites. J Biol Chem 278: 34700-34708.

Qin, C., R. D’Souza, J.Q. Feng (2007) Dentin matrix protein 1 (DMP1): new and important roles for biomineralization and phosphate homeostasis. J Dent Res 86: 1134-1141.

Qin, C., B. Huang, J.N. Wygant, B.W. McIntyre, C.H. McDonald, R.G. Cook, W.T. Butler (2006) A chondroitin sulfate chain attached to the bone dentin matrix protein $1 \mathrm{NH}_{2}$-ter- minal fragment. J Biol Chem 281: 80348040.

Steiglitz BM, M. Ayala, K. Narayanan, A. George, D.S. Greenspan DS (2004) Bone morphogenetic protein-1/Tolloid-like proteinases process dentin matrix protein-1. J Biol Chem 279: 980-986.

Sun, Y., V. Gandhi, M. Prasad, W. Yu, X. Wang, Q.Zhu, J.Q. Feng, R.J. Hinton, C. Qin (2010a) Distribution of small integrin-binding ligand, N-linked glycoproteins (SIBLING) in the condylar cartilage of rat mandible. Int J Oral Maxillofac Surg 39: 272-281.

Sun, Y., S. Ma, J. Zhou, A. Yamoah, J.Q. Feng, R.J. Hinton, C. Qin (2010b) Distribution of small integrin-binding ligand, N-linked glycoproteins (SIBLING) in the articular cartilage of the rat femoral head. J Histochem Cytochem 58: 1033-1043

Sun, Y., M. Prasad, T. Gao, X. Wang, Q. Zhu, R. D’Souza, J.Q. Feng, C. Qin (2010c) Failure to process dentin matrix protein 1 (DMP1) into fragments leads to its loss of function in osteogenesis. J Biol Chem 285: 31713-31722.

Tartaix, P.H., M. Doulaverakis, A. George, L.W. Fisher, W.T. Butler, C. Qin, E. Salih, M. Tan, Y. Fujimoto, L. Spevak, A.L. Boskey (2004) In vitro effects of dentin matrix protein-1 on hydroxyapatite formation provide insights into in vivo functions. J Biol Chem 279: 18115-18120

Ye, L., M. MacDougall, S. Zhang, Y. Xie, J. Zhang, Z. Li, Y. Lu, Y. Mishina, J.Q. Feng (2004) Deletion of dentin matrix protein-1 leads to a partial failure of maturation of predentin into dentin, hypomineralization, and expanded cavities of pulp and root canal during postnatal tooth development. J Biol Chem 279: 19141-19148.

Ye, L., Y. Mishina, D. Chen, H. Huang, S.L. Dallas, M.R. Dallas, P. Sivakumar, T. Kunieda, T.W. Tsutsui, A. Boskey, L.F. Bonewald, J.Q. Feng (2005) Dmp1-deficient mice display severe defects in cartilage formation responsible for a chondrodysplasia-like phenotype. J Biol Chem 280: 6197-6203. 